\title{
Serologic Abnormalities in Macular Lymphocytic Arteritis with Case Presentation
}

\author{
Nicole R. Bender Elizabeth L. Bisbee Douglas Robins Kiran Motaparthi \\ Vladimir Vincek
}

Dermatology, University of Florida, Gainesville, FL, USA

\section{Keywords}

Macular lymphocytic arteritis - Lymphocytic thrombophilic arteritis - Serologic abnormalities .

Antiphospholipid antibodies · Lupus autoantibodies

\begin{abstract}
Macular lymphocytic arteritis (MLA) is an indolent cutaneous small-medium-vessel vasculitis characterized by widespread asymptomatic livedo racemosa. A number of serologic abnormalities have been reported including an elevated erythrocyte sedimentation rate and antibodies associated with antiphospholipid antibody syndrome. We present a case of MLA with multiple serologic abnormalities, including those that have yet to be reported, such as anti-U1 ribonucleotide protein, anti-RNA polymerase III, anti-smith, and anti-proteinase 3 antibodies. We also provide a brief review of this unfamiliar entity with a focus on the appropriate workup.
\end{abstract}

(C) 2022 The Author(s).

Published by S. Karger AG, Basel

\section{Introduction}

Macular lymphocytic arteritis (MLA), first described by Fein etal. [1] in 2003, is a rare mediumvessel vasculitis affecting the skin. This diagnosis has also been described under a different name, lymphocytic thrombophilic arteritis (LTA), to better emphasize the histologic features [2]. There has been debate regarding whether MLA and LTA represent distinct diagnoses from each other, as well as if they lie on a spectrum with cutaneous polyarteritis nodosa (cPAN), representing an early or indolent form of the disease [3]. Along with the majority of publications, we will presume MLA and LTA represent the same diagnosis, which exhibits minor clinical variations. 
Many patients will have an abnormal serologic examination, including an elevated erythrocyte sedimentation rate (ESR), positiveanticardiolipin (ACL) antibody, and antinuclear antibody profiles [4]. Despite these studies, the disease is overwhelmingly confined to the skin, and thus far long term follow up has not identified progression to a systemic vasculitis or cPAN [5]. We describe a case of MLA associated with multiple persistent positive autoantibody profiles that have not yet been previously reported.

\section{Case Report/Case Presentation}

A 48-year-old man from Trinidad and Tobago presented to the dermatology clinic with a 1-year history of asymptomatic hyperpigmentation on the bilateral lower legs and arms, mid chest, and abdomen. He had previously been found to have a number of abnormal serologic studies including an elevated ESR, positive ACL IgM, antinuclear antibody, anti-U1 ribonucleotide protein, anti-RNA polymerase III, anti-Smith, and anti-proteinase 3 antibodies. The full serologic assessment is shown in Table 1 . The patient did not meet accepted criteria for systemic lupus erythematosus, scleroderma, or mixed connective tissue disorder.

On physical exam, the patient had ill-defined dark brown hyperpigmented patches in a reticulate pattern resembling livedo racemosa on the lower legs (shown in Fig. 1) and an annular pattern on the mid chest. He had faint ill-defined brown macules and patches on the abdomen and arms. The remainder of his physical exam was normal, and full review of systems was negative for systemic symptoms. Histopathology from the left lower leg demonstrated a medium-vessel vasculitis involving a vessel at the deep dermal-subcuticular junction (shown in Fig. 2). The inflammatory infiltrate was composed almost entirely of lymphocytes with very rare neutrophils. There was luminal fibrin deposition and narrowing of the vessel lumen.

Systemic workup, including CBC, CMP, creatine kinase, and aldolase, thyroid studies, urinalysis, and transthoracic echocardiogram were normal with the exception of a mildly decreased leukocyte count at 3.5 thou $/ \mathrm{mm}^{3}$ and mild to moderate tricuspid regurgitation. Given the asymptomatic nature of the eruption and that there currently is no established effective treatment for MLA, the patient elected for clinical monitoring.

\section{Discussion}

The clinical features of MLA have been well recognized as asymptomatic livedo racemosa or pigmented macules predominantly located on the lower legs. In most reports, these lesions are non-indurated, and nodules or ulcerations are absent on physical exam. Atypical clinical presentations including annular morphology, unilateral or diffuse distribution of lesions, the presence of nodules, ulcerations, or systemic involvement such as testicular infarction and neuropathy have been reported [6-10]. MLA disproportionately affects women, primarily of black ethnicity, with the second most common groups being Asian and Caucasian patients [4]. Histologically, there is a lymphocyte-predominant small-to-medium sized vessel arteritis, affecting vessels in the deep dermis or upper subcutis. Neutrophils are absent to scarce, and the arteries are typically narrowed or occluded. Fibrin deposition within the vessel wall forming a distinct luminal ring is a hallmark of MLA [11]. LTA has been separately described, but has similar clinical and histologic features to MLA, with occasional infiltrative papules, nodules or ulcers [2]. In general, MLA and LTA are considered the same disease with minor variations in their clinical presentation.

Additional confusion regarding whether these entities lie on a spectrum with cutaneous polyarteritis nodosa (cPAN) exists due to overlapping clinical and histologic features. A recent

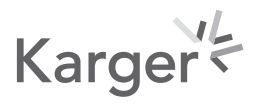


Table 1. Serologic assessment in our patient with MLA

\begin{tabular}{|c|c|c|}
\hline Lab & Measurement & Normal reference range \\
\hline $\mathrm{ESR}, \mathrm{mm} / \mathrm{h}$ & 33 & $0-10$ \\
\hline $\mathrm{CRP}, \mathrm{mg} / \mathrm{L}$ & 0.62 & $0.20-5.00$ \\
\hline ACL IgM, mpl $\mu / \mathrm{mL}$ & 30.3 & $<20$ \\
\hline ACL IgG, gpl $\mu / \mathrm{mL}$ & $<9.4$ & $<20$ \\
\hline Anti-ß2 glycoprotein I IgM, smu $\mu / \mathrm{mL}$ & $<9.4$ & $<20$ \\
\hline Anti-ß2 glycoprotein I IgG, sgu $\mu / \mathrm{mL}$ & $<9.4$ & $<20$ \\
\hline Lupus anticoagulant & Negative & \\
\hline ANA & 1:1,280 speckled & $<1: 40$ \\
\hline Anti-Smith (AI) & $>8.0$ & $0.0-0.9$ \\
\hline Anti-centromere (AI) & $<0.2$ & $0.0-0.9$ \\
\hline Anti-dsDNA, IU/mL & $<1$ & $0-9$ \\
\hline SSA-Ro (AI) & $<0.2$ & $0.0-0.9$ \\
\hline SSB-La (AI) & 0.2 & $0.0-0.9$ \\
\hline Anti-Scl-70 (units) & $<20$ & $<20$ \\
\hline Anti-PM/Scl-100 (units) & $<20$ & $<20$ \\
\hline Anti-PM/Scl-75 (units) & $<20$ & $<20$ \\
\hline RNP (AI) & $>8.0$ & $0.0-0.9$ \\
\hline Anti-U1 RNP (units) & 25 & $<20$ \\
\hline Anti-U3 RNP/fibrillarin & Negative & \\
\hline Anti-RNA polymerase III (units) & 27 & $<20$ \\
\hline c-ANCA & $<1: 20$ & $<1: 20$ \\
\hline p-ANCA & $<1: 20$ & $<1: 20$ \\
\hline Anti-proteinase $3, \mathrm{U} / \mathrm{mL}$ & 4.5 & $0.0-3.5$ \\
\hline Atypical p-ANCA & $<1: 20$ & $<1: 20$ \\
\hline $\mathrm{RF}, \mathrm{IU} / \mathrm{mL}$ & $<10$ & $0.0-14.0$ \\
\hline Anti-CCP IgG, units & 8 & $0-19$ \\
\hline $\mathrm{C} 3, \mathrm{mg} / \mathrm{dL}$ & 118 & $82-167$ \\
\hline $\mathrm{C} 4, \mathrm{mg} / \mathrm{dL}$ & 23 & $12-38$ \\
\hline Total CK, U/L & 244 & $49-439$ \\
\hline Aldolase, U/L & 3.8 & $3.3-10.3$ \\
\hline
\end{tabular}

CRP, c-reactive protein; ANA, antinuclear antibody; RNP, ribonucleotide protein; ANCA, anti-neutrophil cytoplasmic antibody; $\mathrm{RF}$, rheumatoid factor; $\mathrm{CCP}$, cyclic citrullinated peptide; $\mathrm{C} 3$, complement 3; $\mathrm{C} 4$, complement 4; CK, creatine kinase.

retrospective study compared blinded assessments of the clinicopathologic features of patients diagnosed with LTA and CPAN and determined the discriminating characteristics to be a widespread pattern of non-infiltrated and asymptomatic livedo racemosa with a paucity $(\leq 5 \%)$ of neutrophils on histology for LTA. CPAN, in contrast, was associated with localized starburst livedo, purpura, episodes of pain, nodules and inflammatory ulcerations, and the presence $(\geq 5 \%)$ of neutrophils on histology [6]. While there has been a single report of a patient diagnosed as MLA with evidence of systemic vasculitis (testicular infarcts and mononeuritis) [9], this disease overall follows a persistent indolent course. There have also been 




Fig. 1. Clinical image. Reticulated dark brown macules and patches resembling livedo racemosa on the bilateral lower legs.

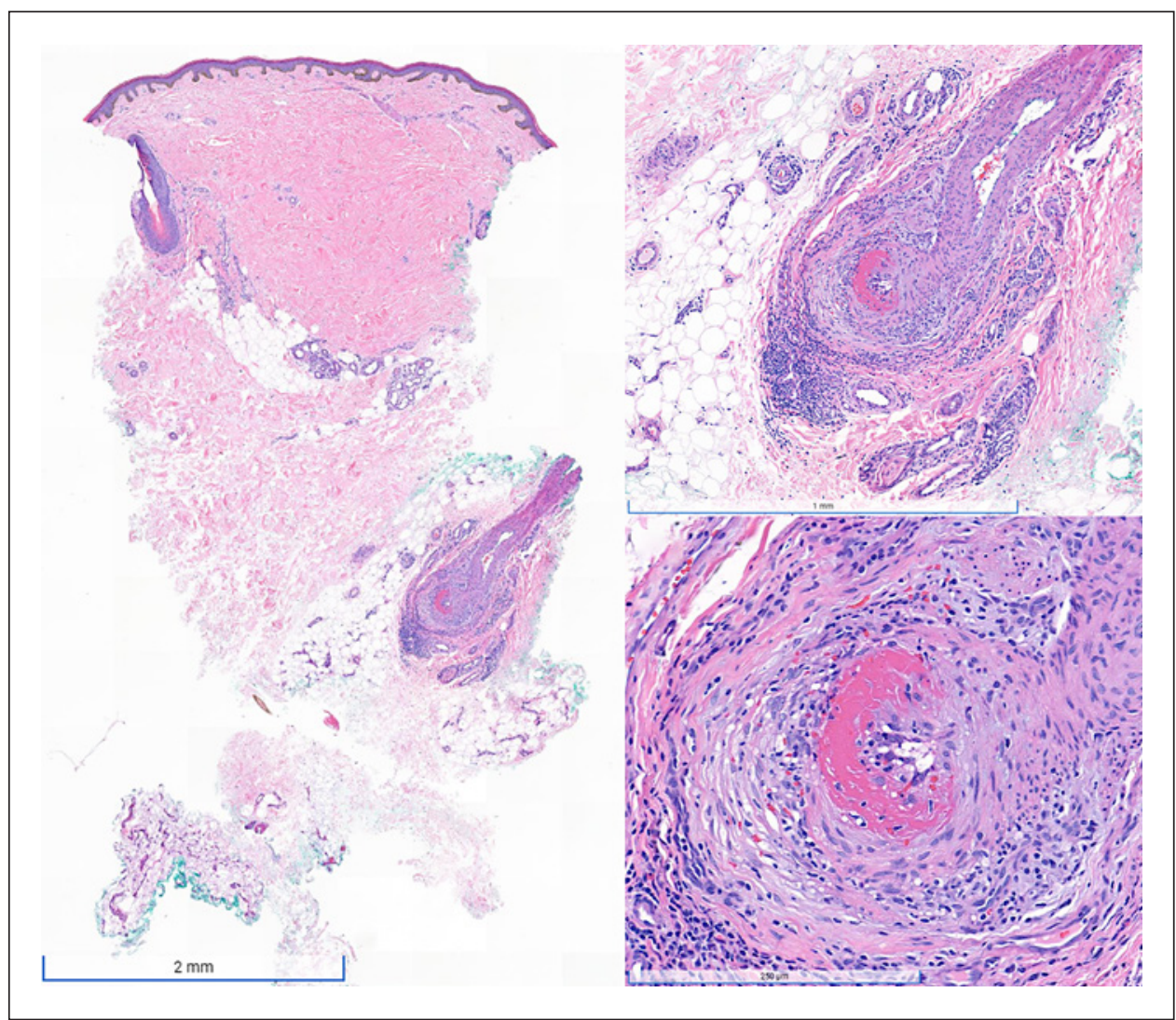

Fig. 2. Histopathology image - punch biopsy from the left lower leg. a H\&E staining $(\times 15)$ demonstrates a medium-vessel vasculitis involving a vessel at the deep dermal-subcuticular junction. b H\&E staining $(\times 40)$ demonstrates lymphocytes with very rare neutrophils in the inflammatory infiltrate with luminal fibrin deposition and narrowing of the vessel lumen. $\mathbf{c H} \& \mathrm{E}$ staining $(\times 100)$ demonstrates a distinct luminal ring of fibrin within the affected vessel. H\&E, hematoxylin and eosin. 
Case Reports in Dermatology

Table 2. Reported lab abnormalities in patients with MLA

\begin{tabular}{l|l}
\hline Case Rep Dermatol 2022;14:12-18 \\
\hline DOI: 10.1159/000519658 & $\begin{array}{l}\text { @ 2022 The Author(s). Published by S. Karger AG, Basel } \\
\text { www.karger.com/cde }\end{array}$ \\
\hline
\end{tabular}

Bender et al.: Serologic Abnormalities in Macular Lymphocytic Arteritis

\begin{tabular}{ll}
\hline Lab & $\begin{array}{l}\text { Patients reported, } n \\
\text { (including our patient) }\end{array}$ \\
\hline $\begin{array}{l}\text { Inflammatory markers } \\
\text { ESR }\end{array}$ & 12 \\
Autoantibodies & \\
ANA & 20 \\
ACL antibody & 12 \\
Anti- $\beta 2$ glycoprotein & 4 \\
Lupus anticoagulant & 4 \\
RF & 2 \\
ANCA & 4 (3 unspecified, 1 c-ANCA) \\
Anti-Proteinase 3 & 1 \\
SSA-Ro & 1 \\
Anti-histone & 1 \\
Anti-RNP antibodies & 1 \\
Anti-RNA polymerase III & 1 \\
Anti-CCP & 1 \\
Prothrombotic factors & \\
Antiphospholipid antibodies (as above) & 20 \\
Factor V Leiden gene mutation & 4 (heterozygous) \\
Prothrombin gene mutation & 3 (heterozygous) \\
Low protein S activity & 1 \\
Infectious studies & \\
HIV & 2 \\
HBV & 1 \\
\hline
\end{tabular}

ANA, antinuclear antibody; RF, rheumatoid factor; ANCA, antineutrophil cytoplasmic antibody; RNP, ribonucleotide protein; CCP, cyclic citrullinated peptide; HIV, human immunodeficiency virus; HBV, hepatitis B virus.

no reports of MLA/LTA with progression to cPAN, which also supports consideration of MLA/ LTA and cPAN as distinct entities.

Epidemiologically MLA is frequently seen in women, particularly women of color, with roughly one third of reported patients being of African descent [4]. MLA also tends to affect patients at a younger age compared to cPAN and has been associated with both human immunodeficiency virus and hepatitis B [12]. Interestingly, MLA has been associated with a number of lab abnormalities, shown in Table 2, including an elevated ESR, and positive ACL, anti- $\beta 2$ glycoprotein, and antinuclear antibodies. Despite these findings, reported patients fail to meet criteria for specific autoimmune conditions, and occasionally these abnormalities are transitory with reversion back to normal in the months following diagnosis.

In addition to positive antiphospholipid antibodies, there have been reports of patients with heterozygous mutations in Factor V Leiden and prothrombin genes. While these generally confer risk for hypercoagulable states, end-organ damage from thromboembolic changes have not been reported in patients with MLA/LTA. This includes patients with other risk factors such as smoking and pregnancy. Possible exceptions to this include the single report 
of systemic involvement with testicular infarction [9] and 1 patient who developed transient vision loss in 1 eye lasting 2 min [13]. Criteria for the diagnosis of MLA/LTA has been posed as: (1) presence of macules, papules, and/or patches that follow a benign course; (2) histopathology demonstrating small-medium-vessel vasculitis with a predominantly lymphocytic infiltrate; and (3) absence of signs or symptoms to suggest systemic vasculitis [4].

Our patient presented with both typical clinical and histopathological characteristics of MLA and met the criteria stated above. He also had a number of serologic abnormalities including those known to be associated with MLA such as an elevated ESR and positive ACL and antinuclear antibodies, as well as those that have yet to be reported including anti-U1 ribonucleotide protein, anti-RNA polymerase III, anti-smith, and anti-proteinase 3 antibodies. Our patient, however, fails to meet criteria for any specific rheumatologic disease. It had previously been shown that $17 \%$ of patients with primary systemic vasculitis had autoantibodies associated with antiphospholipid antibody syndrome on at least 1 occasion [2]. While it is theorized that these autoantibodies are produced following exposure to antigens found on injured endothelial cells from vascular destruction, this does not explain the association of MLA with rheumatoid factor, anti-neutrophil cytoplasmic antibody, or lupus antibodies (anti-smith, anti-histone, and anti-dsDNA).

No effective treatment has been identified thus far for MLA. Treatments that have been attempted include anti-inflammatory or immunomodulators such as oral and topical steroids, methotrexate, hydroxychloroquine, colchicine, dapsone, pentoxyifylline, and diclofenac, as well as anticoagulants including aspirin, clopidogrel, warfarin, and low molecular weight heparin. Other miscellaneous treatments included nifedipine, doxycycline, and compression stockings. The majority of patients are managed expectantly given the asymptomatic and indolent nature of the disease [4].

\section{Conclusion}

Since MLA was first reported over 15 years ago, there have been over 50 cases described in the literature [6]. However, common knowledge and familiarity with this entity are generally lacking in both the dermatology and pathology fields. The true incidence of this disorder is likely not known, as patients with darker skin tones presenting with hyperpigmentation may simply be dismissed as post-inflammatory hyperpigmentation. Therefore, it is paramount to continue to define MLA/LTA, identify effective treatment options, and include this entity in continuing medical education and trainee didactics. There currently is no established recommended systemic workup or treatment. Given the indolent course, further laboratory testing is not necessary.

\section{Statement of Ethics}

Written informed consent was obtained from the patient for publication of this case report and any accompanying images. Every precaution has been taken to protect the privacy of research subjects and confidentiality of their personal information. This publication complies with the guidelines for human studies and was conducted ethically in accordance with the World Medical Association Declaration of Helsinki.

\section{Conflict of Interest Statement}

The authors have no conflicts of interest to declare.

\section{Karger'}




\section{Funding Sources}

The authors have no funding sources to declare.

\section{Author Contributions}

Nicole R. Bender reviewed the histopathology of the patient and performed search and analysis of information, initial drafting of the manuscript, and final approval of the manuscript. Elizabeth Bisbee and Douglas Robins aided in the acquisition of medical data including clinical examination, critical revision of the manuscript, and final approval of the manuscript. Vladimir Vincek and Kiran Motaparthi reviewed the histopathology of the patient, confirmed the diagnosis, conceptualized the project, performed critical revision of the manuscript, and final revision/approval of the manuscript.

\section{Data Availability Statement}

All data generated or analyzed in this case report are included in this article. Further inquiries can be directed to the corresponding author.

\section{References}

1 Fein $\mathrm{H}$, Sheth AP, Mutasim DF. Cutaneous arteritis presenting with hyperpigmented macules: macular arteritis. J Am Acad Dermatol. 2003 Sep;49(3):519-22.

2 Lee JS, Kossard S, McGrath MA. Lymphocytic thrombophilic arteritis: a newly described medium-sized vessel arteritis of the skin. Arch Dermatol. 2008 Sep;144(9):1175-82.

3 Macarenco RS, Galan A, Simoni PM, Macarenco AC, Tintle SJ, Rose R, et al. Cutaneous lymphocytic thrombophilic (macular) arteritis: a distinct entity or an indolent (reparative) stage of cutaneous polyarteritis nodosa? Report of 2 cases of cutaneous arteritis and review of the literature. Am J Dermatopathol. 2013 Apr;35(2):213-9.

4 Vakili S, Zampella JG, Kwatra SG, Blanck J, Loss M. Lymphocytic thrombophilic arteritis: a review. J Clin Rheumatol. 2019 Apr;25(3):147-52.

5 Morita TCAB, Trés GFS, Criado PR. Is macular lymphocytic arteritis limited to the skin? Long-term follow-up of seven patients. An Bras Dermatol. 2020 Jan;95(1):32-9.

6 Kelly RI, Wee E, Balta S, Williams RA. Lymphocytic thrombophilic arteritis and cutaneous polyarteritis nodosa: clinicopathologic comparison with blinded histologic assessment. J Am Acad Dermatol. 2020 Aug;83(2):501-8.

7 Arana-Guajardo A, Mendoza-Rodríguez C, Miranda-Maldonado I. Unilateral macular lymphocytic arteritis. J Rheumatol. 2016 Sep;43(9):1763-4.

8 Llamas-Velasco M, García-Martín P, Sánchez-Pérez J, Sotomayor E, Fraga J, García-Diez A. Macular lymphocytic arteritis: first clinical presentation with ulcers. J Cutan Pathol. 2013 Apr;40(4):424-7.

9 Wee E, Nikpour M, Balta S, Williams RA, Kelly RI. Lymphocytic thrombophilic arteritis complicated by systemic involvement. Australas J Dermatol. 2018 Aug;59(3):223-5.

10 Shen S, Williams RA, Kelly RI. Neuropathy in a patient with lymphocytic thrombophilic arteritis. Australas J Dermatol. 2013;54:e28-32.

11 Buffiere-Morgado A, Battistella M, Vignon-Pennamen M-D, de Masson A, Rybojad M, Petit A, et al. Relationship between cutaneous polyarteritis nodosa (cPAN) and macular lymphocytic arteritis (MLA): blinded histologic assessment of 35 cPAN cases. J Am Acad Dermatol. 2015 Dec;73(6):1013-20.

12 Kolivras A, Thompson C, Metz T, André J. Macular arteritis associated with concurrent HIV and hepatitis B infections: a case report and evidence for a disease spectrum association with cutaneous polyarteritis nodosa. J Cutan Pathol. 2015 Jun;42(6):416-9.

13 Kelly RI, Wee E, Tancharoen C, Tam MM, Balta S, Williams RA. Three cases of lymphocytic thrombophilic arteritis presenting with an annular eruption. Australas J Dermatol. 2018 May;59(2):e127-32. 